\title{
Influence of curing protocols on water sorption and solubility of a self-adhesive resin-cement
}

\author{
Dilcele Silva Moreira Dziedzic ${ }^{1}$, João Paulo Stanislovicz Prohny', Gledson Luiz Picharski², Adilson Yoshio Furuse³ \\ 'Universidade Positivo - UP, School of Dentistry, Area of Dental Material, Curitiba, PR, Brazil \\ ${ }^{2}$ Faculdades Pequeno Príncipe, Department of Biostatistics, Curitiba, PR, Brazil \\ ${ }^{3}$ Universidade de São Paulo - USP, Bauru Dental School, Department of Operative Dentistry, Endodontics and Dental Materials, Bauru, SP, Brazil
}

\begin{abstract}
Aim: To evaluate the effect of different activation protocols on the polymerization of a self-adhesive dual cured resin-based cement. Methods: Thirty disc-shaped specimens were prepared with the resin cement RelyXU200 (3M ESPE) and divided according to three protocols: immediate light-activation for $40 \mathrm{~s}$, delayed light-activation (10 min after manipulation, for $40 \mathrm{~s}$ ) or self-curing without lightactivation. The specimens were desiccated, kept in water at $37^{\circ} \mathrm{C}$ for 7 days and desiccated again to calculate water sorption, solubility and mass variation. Data were analyzed by Shapiro-Wilk Test and Wilcoxon tests $(\alpha=0.05)$. Images after the specimens' final desiccation were also made. Results: The Wilcoxon test revealed a significant difference for sorption and mass variation $(p<0.05)$ and the highest value was observed in self-curing or chemical activation group (CA), followed by delayed light-activation (DL) and immediate light-activation (IL). Besides the water sorption parameters, there were also microvoids on the discs from the delayed and no light-activation groups. Conclusions: The light-activation immediately after manipulation is recommended for the evaluated resin cement.
\end{abstract}

Keywords: Resin Cements. Solubility. Polymerization.

\section{Introduction}

The degree of conversion and polymerization rate of dual-curing resin-based cements can determine the longevity of the indirect restoration, because it is directly related to the materials' properties. Besides the bonding mechanisms to both dental tissues and restorations, the success of luting cements depends on the mechanical properties developed during polymerization to be preserved during clinical life. Therefore, all involved procedures and the used materials for cementation of indirect

Received for publication: August 08, 2016 Accepted: November 01, 2016

Correspondence to: Adilson Yoshio Furuse Departamento de Dentística, Endodontia e Materiais Odontológicos Faculdade de Odontologia de Bauru Universidade de São Paulo

Al. Octávio Pinheiro Brisolla, 9-75, Bauru São Paulo, Brasil - CEP: 17012-901 Phone: +55 14 3235-8253 E-mail: furuse@usp.br restorations and intracanal posts are critical to achieving adequate performance of indirect restorations.

Dual-curing resin cements present two activation systems (chemical and light activation) for a thorough and uniform conversion of monomers into polymers, even when the composition, thickness and color of the ceramic restoration could hamper the transmission of the curing light used to polymerize the cement1. Thus, different light-activation protocols should be investigated, as the most significant effects on the properties of resin cements are related to the type of cement, exposure time, followed by translucency, ceramic type and thickness of the ceramic restoration ${ }^{1-3}$.

The effect of light activation on dual-cured resin cements should not be neglected for some cements ${ }^{4-7}$. However, the immediate light-activation of a dual-cured resin- 
cement, as recommended by the manufacturer, may have a deleterious effect on polymerization if it stiffens the polymer, limiting the process of chemical activation. This prevents the cement from achieving its maximum degree of conversion and mechanical properties ${ }^{8}$. The light-activation delay to allow more time for the chemical activation to occur before light-activation seems to affect the dual-cured resin cement properties depending on the material's composition and the time interval between the mixture until light-activation. Light-activation delay of $5 \mathrm{~min}$, was suggested as being advantageous to reduce shrinkage stress during polymerization, without impairing the degree of conversion, the extent of polymerization and hardness of some dual-cured cements $^{9-11}$. It was also demonstrated that a 6-minute delay after the manipulation of resin cements may result in increased color stability $^{2}$, but a 10-minute delay may be harmful ${ }^{12}$.

During polymerization, individual polymer chains are formed by covalent bonds, which are crosslinked by secondary bonds. The degree of conversion of the material, which is the average number of monomers that form the polymer chains, is directly related to the length and crosslinking of the network and indirectly to water sorption. The polymer network of a resin-based material is composed by crosslinked molecules and unreacted monomers not linked to the chains. Inadequate polymerization, in addition to reducing the mechanical properties ${ }^{10,13}$, may have biological consequences $^{14,15}$ related to the unreacted free monomers. ${ }^{16}$ Water sorption and hydrogen bonds with the polar groups of polymers produce separation of the molecules and reduction of hydrogen bonds between them ${ }^{17}$. The expansion resulting from water sorption favours the release of the monomers not linked into chains, reduction of mechanical properties ${ }^{18}$ and the cement plasticisation can generate unsupported areas, causing fracture to indirect restorations ${ }^{19}$.

Sorption and solubility tests are used for in vitro evaluation of resin-based materials ${ }^{20}$, including dual-cured resin cements ${ }^{12}$, to evaluate and recommend the materials with lower sorption and solubility for indirect restorations ${ }^{21}$. The water absorption measured by sorption, influences negatively the material, causing expansion, weakening of matrix and filler bonding, discoloration and reduction of mechanical properties ${ }^{20}$. The solubility of the material reflects the amount of released residual monomers, revealed by its mass reduction ${ }^{20}$. The high water sorption and solubility influence negatively the resinous materials, indicating lower chemical stability, lower biocompatibility, higher expansion and lower mechanical properties. Sorption, solubility and mass variation are considered indicators of the chemical, physical and mechanical properties observed in the structure of the polymeric network $^{12,21}$. Alterations of these properties can lead to deleterious clinical effects. Therefore, parameters of sorption, solubility and mass variation can be used to demonstrate the incompatibility of the material for certain clinical applications.

Dual-curing resin cements of different brands showed different behaviours as to the degree of conversion and mechanical properties when cured with different light-activation protocols ${ }^{22-24}$. While some cements are more suitable for situations where immediate light-activation is possible, others show a better behaviour when the light-activation is limited ${ }^{23}$. The mechanical properties of cements are mostly affected by their composition rather than the used activation mode and the delay of $5 \mathrm{~min}$ for the polymerization did not interfere with the assessed properties ${ }^{22}$. The period of $5 \mathrm{~min}$ for chemical conversion can even improve the clinical performance of restorations by reducing shrinkage stress without interfering with the degree of conversion'.

The aim of the present study was to evaluate sorption (S), solubility (SL) and mass variation (M\%) of the dual-cured selfcuring cement RelyX U200 (3M ESPE) when three different polymerization protocols were used. The protocols were: immediate light-activation, $10 \mathrm{~min}$ delayed light-activation and self-activation. The tested null hypothesis was that there was no difference between the polymerization protocols for the values of each parameter: S, SL and M\%.

\section{Material and methods}

Thirty disc-shaped specimens were prepared with the dual-cured self-adhesive resin cement (RelyX U200; 3M ESPE Deutschland GmbH, Neuss, Germany, colour A3 Opaque, Lot: 506385). The main components of the cement, presented by weight in the Material Safety Data Sheet, are silane treated glass powder $(65 \%)$ and substituted dimethacrylate $(30 \%)$ in the catalyst paste; silane treated glass powder (45-55\%), methylesterdimethacrylate acid (20-30\%), triethylene glycol dimethacrylate (10-20\%), and silane treated silica $(10 \%)$ in the base paste. Both pastes also contain initiator components, stabilizers and rheological additives. The two pastes (1:1 ratio) were hand mixed on a mixing pad for $10 \mathrm{~s}$ and placed into a circular nylon mould (1 mm thick with a central orifice with $7 \mathrm{~mm}$ diameter), between two Mylar strips and two 1-mm-thick glass slides. In order to obtain parallel surfaces, light finger pressure was applied on the top glass slide. The cement was light-activated on the top exposed surface in continuous mode for $40 \mathrm{~s}$ with a LED curing device (Wireless Poly; Kavo, Joinville, $\mathrm{SC}$, Brazil) at $1,100 \mathrm{~mW} / \mathrm{cm}^{2}$.

Three groups were evaluated according to the light-activation protocol: immediate light-activation, delayed light-activation or chemical activation. The immediate light-activation (IL) was done approximately $1 \mathrm{~min}$ after specimen preparation. In the delayed light-activation group (DL), after manipulation of the cement, the samples were protected from light for $10 \mathrm{~min}$ before light-activation. The light-activation of these groups was initially conducted through a $1 \mathrm{~mm}$-thick glass plate, which was then removed and the process continued through the Mylar strip. Chemical activation (CA) or self-curing specimens were prepared, covered with black plastic and away from light exposure for 24 h, with no light-activation. Each resin cement disc was visually inspected to exclude those with porosities. Excess material at the edges was removed with a \#15 scalpel blade and the borders were smoothed with 600-grit sandpaper. Discs were stored in amber glass bottles and retained from any light for $24 \mathrm{~h}$ at room temperature.

The discs were washed with distilled water in an ultrasonic cleaner (Cristófoli, Campo Mourão, PR, Brazil) for 2 min and dried with absorbent paper before desiccation. Dark plastic canisters with calcium chloride and the amber glass vials containing the specimens were placed in a desiccator with silica and under 
vacuum suction (400 $\mathrm{mmHg}$ ) for $2 \mathrm{~h}$ at room temperature. The vials, kept in desiccation and dark conditions, were transferred to an incubator (Labstore Equipment, Curitiba, PR, Brazil) at $37{ }^{\circ} \mathrm{C}$ for $24 \mathrm{~h}$. The specimens were weighed every $24 \mathrm{~h}$ on an analytical scale (Boeco, Hamburg, Germany) with a $\pm 0.01 \mathrm{mg}$ accuracy to obtain a constant mass $(\mathrm{ml})$, with a variation lower than $0.2 \mathrm{mg}$. The thickness and the diameter of the discs were measured using a digital calliper (Starret 799 A-6/150, Itu, SP, Brazil), at four places around the circumference, in order to calculate the volume of the specimen $(\mathrm{V}$, in $\mathrm{mm} 3)$ with the following equation:

$$
\mathrm{V}=\pi r^{2} \mathrm{~h}
$$

Where $r$ is the mean radius of the specimen, and $h$ is the average thickness.

The specimens were kept in amber glass bottles containing $10 \mathrm{~mL}$ distilled water ( $\mathrm{pH} \mathrm{7.2)} \mathrm{for} 7$ days, sealed at $37 \mathrm{C}$. The water was changed every $48 \mathrm{~h}$ during this period. The samples were dried with absorbent paper and weighed again $(\mathrm{m} 2)$ before a second desiccation cycle, as described previously, to obtain the final mass (m3).

The water sorption (S) and solubility (SL) were calculated by the following equations, respectively:

$$
\begin{aligned}
& \mathrm{S}=(\mathrm{m} 2-\mathrm{m} 3) / \mathrm{V} \\
& \mathrm{SL}=(\mathrm{m} 1-\mathrm{m} 3) / \mathrm{V}
\end{aligned}
$$

Where $\mathrm{m} 1$ is the initial mass of the desiccated specimen before immersion in distilled water, $\mathrm{m} 2$ is the mass of saturated sample after immersion in water for 7 days, $\mathrm{m} 3$ is the final mass after desiccation, and $\mathrm{V}$ is the volume of sample.

The percent change in mass ( $\mathrm{M} \%)$ was calculated by the following equation:

$$
\mathrm{M} \%=(\mathrm{m} 2-\mathrm{m} 1) / \mathrm{m} 1 \times 100
$$

The data for water sorption (S), solubility (SL) and percentage change in mass (M\%) were analyzed by Shapiro-Wilk test and Wilcoxon nonparametric test (R Core Team Software; R Foundation for Statistical Computing, Vienna, Austria), with a $5 \%$ significance level. If the $\mathrm{p}$ value obtained from the test was statistically significant, the group differed from the others. For the purpose of evaluating the surface characteristics like voids, the discs were examined with an optical microscope (Olympus BX41, Tokyo, Japan) at 10x magnification and the images were captured with the Image Pro Plus program.

\section{Results}

Significant differences in water sorption between the protocols used for light-activation were observed and are presented in Tables 1 and 2, and Figures 1 and 2. The Wilcoxon tests revealed highly significant differences among the three activation protocols for mass variation with no exception (Table 2). After storage in water, there was a significant difference between chemical activation and the other two groups, $(\mathrm{p}<0.05)$. A significant difference was also observed between immediate and delayed light-activations, the two lowest values for this parameter (Tables 1 and 2, Figures 1 and 2).

Table 1 - Mean values and standard deviations for water sorption, solubility, and percentage of mass variation of the groups after 7 days of storage in water $\left(37^{\circ} \mathrm{C}\right)$.

\begin{tabular}{lccc}
\hline & $\begin{array}{c}\text { Sorption }(\mu \mathrm{g} / \\
\left.\mathrm{mm}^{3}\right)\end{array}$ & Solubility $\left(\mu \mathrm{g} / \mathrm{mm}^{3}\right)$ & Mass variation (\%) \\
\hline $\begin{array}{l}\text { Immediate light- } \\
\text { activation }\end{array}$ & $28.09(3.77) \mathrm{A}$ & $1.28(3.10) \mathrm{A}$ & $1.49(1.10) \mathrm{A}$ \\
$\begin{array}{l}\text { Delayed light-acti- } \\
\text { vation }\end{array}$ & $32.07(3.36) \mathrm{B}$ & $0.04(1.46) \mathrm{A}$ & $1.74(0.12) \mathrm{B}$ \\
Chemical activation & $37.58(3.55) \mathrm{C}$ & $-0.53(0.75) \mathrm{A}$ & $2.06(0.17) \mathrm{C}$ \\
\hline
\end{tabular}

Water sorption and solubility presented in absolute values $\left(\mu \mathrm{g} / \mathrm{mm}^{3}\right)$, mass variation presented in percent $(\%)$. Different capital letters in columns indicate significant difference $(p<0.05)$.

Table 2 - p values of Wilcoxon tests of water sorption, solubility and percent of mass variation of the groups after 7 days of storage in water $\left(37^{\circ} \mathrm{C}\right)$. Evidence of difference between groups when $\mathrm{p}<0.05$.

\begin{tabular}{lcccccc}
\hline & \multicolumn{2}{c}{ Sorption } & \multicolumn{2}{c}{ Solubility } & \multicolumn{2}{c}{ Mass variation } \\
\hline & Immediate & Chemical & Immediate & Chemical & Immediate & Chemical \\
\hline Chemical & $<0.001$ & - & 0.32 & - & $<0.001$ & - \\
Delayed & 0.009 & 0.008 & 0.79 & 0.79 & $<0.001$ & $<0.001$ \\
\hline
\end{tabular}

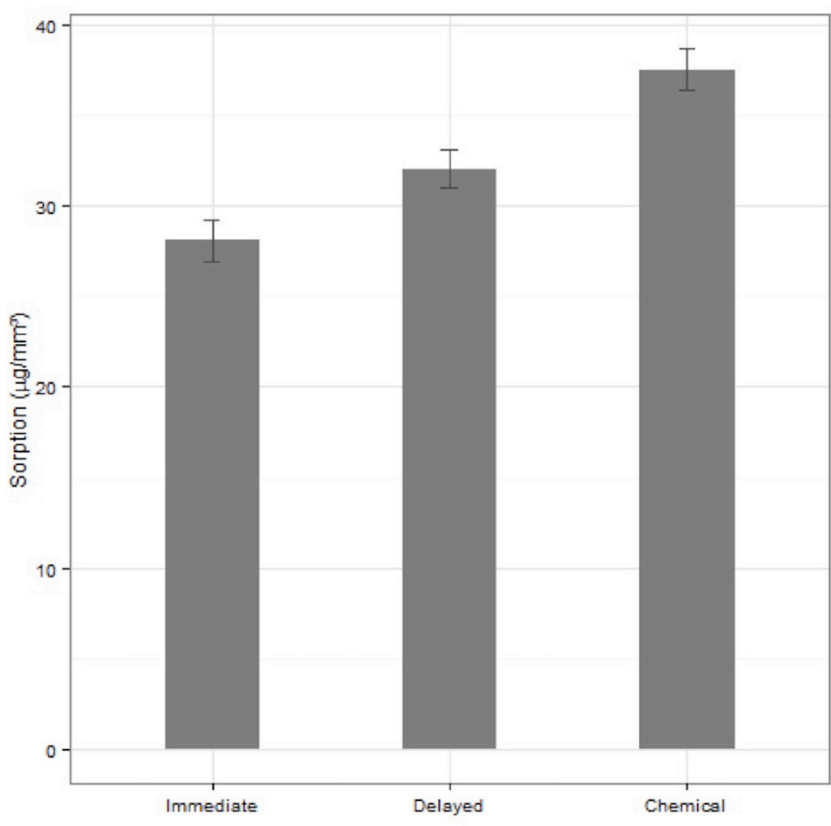

Fig.1. Water sorption characteristics $\left(\mu \mathrm{g} / \mathrm{mm}^{3}\right)$ of the cement under three different activation protocols after seven days of water immersion (mean and standard error) 


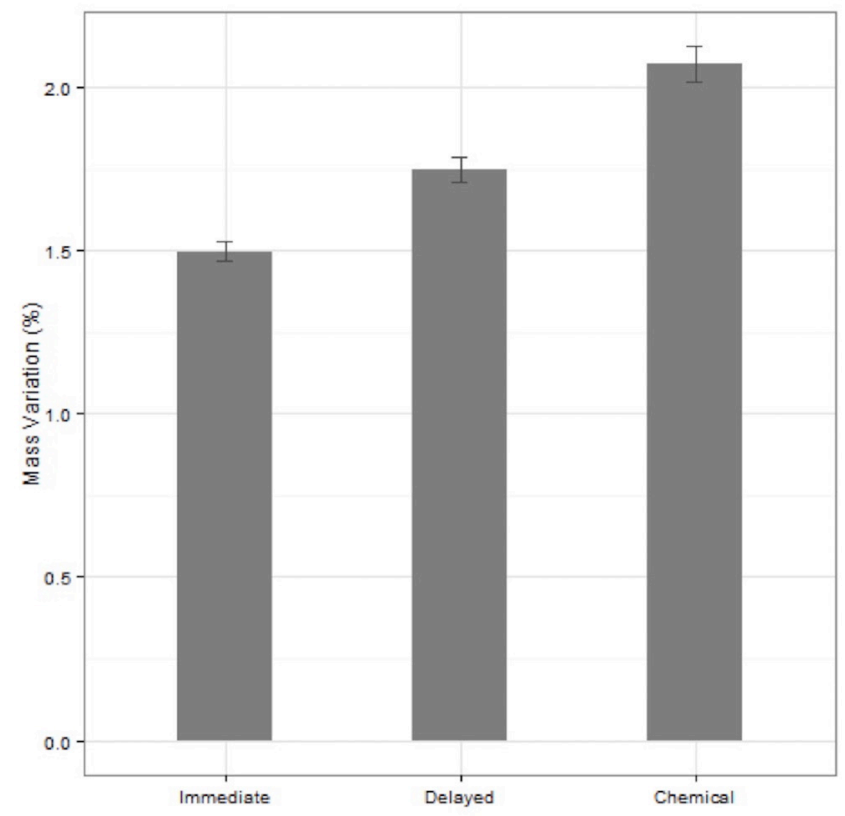

Fig.2. Mass variation percent (\%) of the cement under different activation protocols after seven days of water immersion (mean and standard error).

The solubility, a measure of the amount of unreacted monomer eliminated in the water during 7 days storage at $37^{\circ} \mathrm{C}$, exhibited no evident differences between the activation protocols (Tables 1 and 2). Statistical analysis also expressed significant differences among the three protocols for mass variation $(p<0.001)$. The highest mass variation was observed in CA followed by DL and IL (Figure 2).

Images showed a more homogenous mixture in the immediate light-activation group, and microvoids were observed in the delayed light-activation group and throughout the chemical activation group (Figure 3). There were no cracks in any disc.

\section{Discussion}

In the present study the dual-cured self-adhesive resin cement RelyX U200 was activated according three different curing protocols: IL, DL and CA. Under the experimental conditions, the results demonstrated that these protocols influenced significantly the investigated properties of water sorption and mass variation. Thus, the null hypothesis was rejected. The immediate light-activation significantly decreased water sorption and mass variation. This result may indicate that a higher degree of conversion of this dual-cured self-adhesive cement was attained with immediate light-activation. This means that in clinical practice during cementation procedure the removal of excesses and lightactivation should be performed immediately, since according to the present study a long delay may generate a polymer with inferior properties.

The water storage period of seven days, chosen for the present study, has been extensively studied. Svizero et al. verified water sorption daily for 2 weeks and found that the greatest increase of mass was observed in the first day in water for the cements analysed after three curing protocols ${ }^{12}$. During seven days of storage in water the major components removed from the resinous materials are the residual monomers that have not reacted to form polymeric chains ${ }^{18}$. The highest concentration of eluted monomers in 7 days was also observed by other authors ${ }^{5}$.

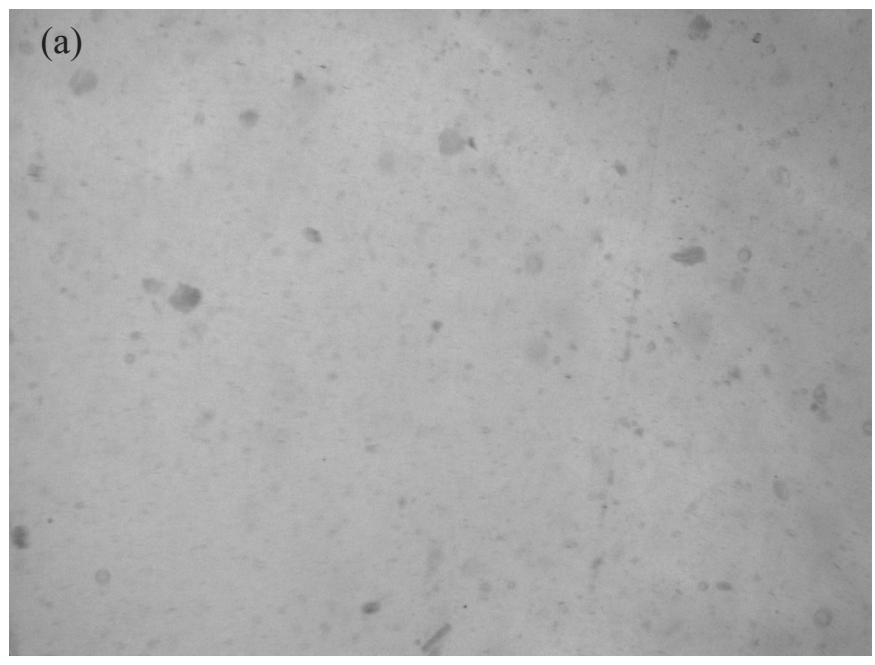

(b)

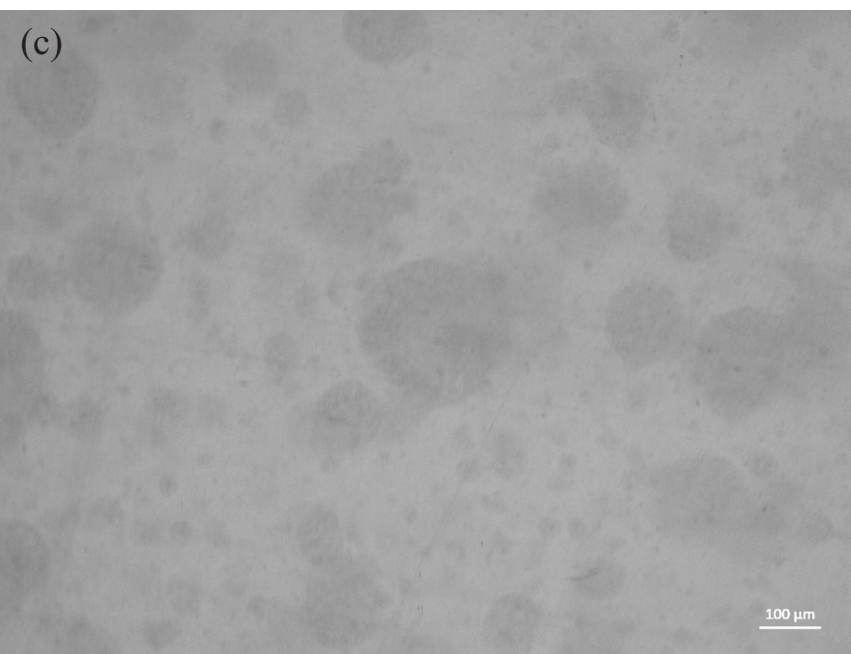

Fig.3. Images of representative discs after the water sorption process (desiccation, water immersion for 7 days and desiccation). (a) The immediate light-activation group showed a more homogenous mixture, and fewer microvoids than the other groups. (b) The delayed light-activated group exhibited microvoids. (c) The chemical activation group showed more and larger microvoids than the others. Field width of each image $=600 \mu \mathrm{m}$. 
Besides the polymerization protocol, sorption and solubility are also explained by the affinity of the organic and inorganic components with water. Hydrophilic monomers and monomers with polar characteristic may account for greater water sorption than hydrophobic monomers ${ }^{5,25}$. The greater density of inorganic filler reduces sorption ${ }^{18}$, but the reaction between filler particles and water can also promote water retention ${ }^{5}$.

The sorption and release of residual monomers are related to the degree of polymerization, the composition of the material: organic matrix and filler particles; and also the solvents used for in vitro studies ${ }^{5,21,25-27}$. Among the factors that affect the hygroscopic and hydrolytic properties of the cements, the degree of polymerization is linked to the sorption, solubility, hydrolytic degradation and the formed polymer, because the quality of the network formed by polymerization regulates the solvent sorption and expansion of the material ${ }^{18}$. Polymer networks with higher density of covalent cross-links have lower absorption and expansion due to the proximity of the polymer chains, reducing the space to be filled by solvent diffusion, as the degree of conversion of the polymer and the pending molecules within the network also influence the absorption of water ${ }^{18}$. Therefore, the polymerization protocols of 10 min-delay and no light-activation may have disturbed the conversion of the dual-cured resin cement producing shorter chains, resulting in significantly higher water sorption. It should be noted that a 10-min delay was proven to be harmful in another study ${ }^{12}$ and it is a time too long for a clinical procedure.

The expansion promoted by the solvent inside the resin matrix, separating the polymer chains, promotes plastification of the material and diffusion of the unreacted monomer, called elution ${ }^{16,18}$. This elution of unreacted monomers during the immersion in water period, results in mass loss and is calculated as solubility. In this study, solubility of the cement under three activation protocols was not significantly different and presented low levels $(\leq 0.1 \%)$, even lower than some reported in the literature ${ }^{12,21}$. The average values of water sorption and solubility of the resin cement RelyX U200 for the three tested polymerization protocols were lower than $40 \mu \mathrm{g} / \mathrm{mm}^{3}$ and $7.5 \mu \mathrm{g} / \mathrm{mm}^{3}$, respectively ${ }^{20}$.

The estimated mean value of solubility for the chemical activation was negative, suggesting that this group of cement, instead of presenting only the expected decrease in mass due to elution of unreacted monomers, showed an increase in mass. Authors observed mass increase in sorption and solubility studies, despite the simultaneous release of free monomers ${ }^{5,25}$. Possible explanations for this increase in mass $(\mathrm{m} 3>\mathrm{m} 1)$ have been suggested: instead of elution of the unreacted monomers, the material retained some of the solvent which was absorbe ${ }^{25}$ and/or the reaction between filler particles and water, with the formation of hydroxides, can result in increased mass ${ }^{5}$.

Studies pointed out that the delay of the light-activation to the maximum tolerable time clinically, from 5 to $10 \mathrm{~min}$, offers an interval for conversion only by chemical activation, which is followed by light-activation to ensure the highest possible degree of conversion ${ }^{8,19,23}$. This study investigated the possibility that lightactivation shortly after mixing the pastes could limit the chemical conversion, preventing a thorough and uniform polymerization, with the different curing protocols. However, the tested $10 \mathrm{~min}$ delay, with the purpose of extending the self-curing reaction, prevented the achievement of the best properties in sorption, solubility and mass variation. Maybe the results could be different if a 5-min-delay was also evaluated.

Inadequate light-activation of dual-cured resin cements may occur under more opaque or thicker ceramic restorations ${ }^{1}$. Therefore, the characteristics in the group without light-activation may be considered clinically relevant. The highest evidence of difference in the values of sorption and mass variation were found in the group with chemical activation compared to the immediate light-activation $(p<0.001)$, where only the polymerization rate of the cement was altered. Therefore, the lowest degree of conversion of the material and/or crosslinking, directly related to water sorption $^{5}$, may explain the higher water sorption of specimens without light-activation.

A possible hypothesis for the higher values of sorption and mass variation in delayed light-activation and no light-activation groups, is that these protocols resulted in polymers with shorter chain length ${ }^{28}$, more sensitive in water sorption probably due to lesser crosslinking, but still with a sufficient degree of conversion to have solubility values as low as the immediate light-activation. According to Marghalani (2012), the percentage of the mass variation or the weight increase of the specimen by absorption of water does not include the free monomers, which are not bonded to the polymer chain and are eliminated simultaneously in water absorption, resulting in reduced mass of the specimen ${ }^{21}$.

It was suggested that the structural parameters of the polymers that affect sorption and solubility are crosslinking density and porosity of the polymer chains ${ }^{25}$. Microscope images showing the density of microvoids in the different groups indicated a good agreement with the numerical data. More microvoids were observed in delayed and no light-activation groups, the ones with higher sorption. Microvoids may be produced by trapped unreacted monomers ${ }^{21}$, enhancing solubility and forming sites for solvent accumulation ${ }^{18,25}$. However, the present study did not use any method to evaluate the degree of conversion. Studies are recommended to confirm the possibility that delay and no lightactivation result in lower molecular weight and crosslink density, causing higher mobility and relaxation between the chains.

Dual-curing resin cements of different brands showed different results as regards the degree of conversion ${ }^{23}$ and mechanical properties ${ }^{22}$, when different polymerization protocols were employed. A comparative study concluded that certain cements are suitable for situations where immediate lightactivation is possible but not in other circumstances where this type of activation could be limited, so that the conversion should not be compromised ${ }^{23}$. Two polymerization protocols employed in this study affected the conversion of the dual-cured self-adhesive cement RelyX U200. However, the immediate light-activation resulted in the best characteristics of significantly lower sorption and mass variation. Further studies should assess the ideal time frame between mixing and light-activation (inferior to $10 \mathrm{~min}$ ) to verify any interference of light-activation with the self-curing mechanism, in order to determine the longest light-activation delay recommended for this cement.

The parameters evaluated in the present study provided the basis for a recommendation on how dentists should use the cement in their daily clinical routine. The lower the sorption, solubility 
and microvoid formation, the better the material in clinical use. Furthermore, the effect of the assembly stability during the delayed light-activation and the chemical activation periods must be known. Two studies describe that finger pressure and $0.5 \mathrm{~kg}$ weight were applied to permit extrusion of excess material ${ }^{13,21}$, while another ${ }^{9}$ used clamps during light-activation delay periods. Most studies do not use any device for stability, with constant support. Even though constant pressure during these periods was not employed by other authors $4-6,10,12,13,22-24,29$, and it is not clinically appropriate, this parameter may have influenced the results, because any loss of stability may favour the formation of microvoids and consequently increase water sorption and solubility.

More research examining other properties may elucidate the effect of light exposure conditions on hardness, degree of conversion and mechanical properties ${ }^{4,30}$. Yan et al. observed that resin cement materials with three different activation modes were cured within $24 \mathrm{~h}$ after mixing or after light activation. The light exposure for light or dual-cured materials produced significantly higher degree of conversion values than the chemically activated ones, compared with the material's initial conversion promoted by light activation ${ }^{30}$. The light activation of dual materials is recommended to produce higher rates of conversion ${ }^{30}$ and hardness ${ }^{4}$, because the relative contributions of the light-cure and chemical-cure mechanisms may differ depending on the material brand.

In conclusion, the alteration of the polymerization protocol from the recommended immediate light-activation to 10-min delay and no light-activation, disturbed the conversion of dual-cured self-adhesive resin cement, resulting in higher water sorption, higher mass variation and presence of microvoids.

\section{Acknowledgements}

This study was carried out during the Undergraduate Research Program at Positivo University, Curitiba, PR, Brazil. The cement employed was kindly supplied by $3 \mathrm{M}$ ESPE.

\section{References}

1. Calgaro PA, Furuse AY, Correr GM, Ornaghi BP, Gonzaga CC. Influence of the interposition of ceramic spacers on the degree of conversion and the hardness of resin cements. Braz Oral Res. 2013;27(5):403-9.

2. Furuse AY, Santana LO, Rizzante FA, Ishikiriama SK, Bombonatti JF, Correr GM, et al. Delayed light activation improves color stability of dual-cured resin cements. J Prosthodont. 2016 Jul 25. doi: 10.1111/ jopr.12509. [Epub ahead of print]

3. Runnacles P, Correr GM, Baratto Filho F, Gonzaga CC, Furuse AY. Degree of conversion of a resin cement light-cured through ceramic veneers of different thicknesses and types. Braz Dent J. 2014;25(1):3842.

4. Braga RR, Cesar PF, Gonzaga CC. Mechanical properties of resin cements with different activation modes. J Oral Rehabil. 2002;29(3):25762.

5. Ortengren $\mathrm{U}$, Wellendorf $\mathrm{H}$, Karlsson $\mathrm{S}$, Ruyter IE. Water sorption and solubility of dental composites and identification of monomers released in an aqueous environment. J Oral Rehabil. 2001;28(12):1106-15.

6. da Silva Fonseca AS, Mizrahi J, Menezes LR, Valente LL, de
Moraes RR, Schneider LF. The Effect of Time between Handling and Photoactivation on Self-Adhesive Resin Cement Properties. J Prosthodont. 2014;23(4):302-7.

7. Kim AR, Jeon YC, Jeong CM, Yun MJ, Choi JW, Kwon YH, et al. Effect of activation modes on the compressive strength, diametral tensile strength and microhardness of dual-cured self-adhesive resin cements. Dent Mater J. 2016;35(2):298-308.

8. Manso AP, Silva NR, Bonfante EA, Pegoraro TA, Dias RA, Carvalho RM. Cements and adhesives for all-ceramic restorations. Dent Clin North Am. 2011;55(2):311-32, ix.

9. Faria-e-Silva A, Boaro L, Braga R, Piva E, Arias V, Martins L. Effect of immediate or delayed light activation on curing kinetics and shrinkage stress of dual-cure resin cements. Oper Dent. 2011;36(2):196-204.

10. Ramos MB, Pegoraro TA, Pegoraro LF, Carvalho RM. Effects of curing protocol and storage time on the micro-hardness of resin cements used to lute fiber-reinforced resin posts. J Appl Oral Sci. 2012;20(5):556-62.

11. Soares CJ, Bicalho AA, Verissimo C, Soares P, Tantbirojn D, Versluis A. Delayed Photo-activation Effects on Mechanical Properties of Dual Cured Resin Cements and Finite Element Analysis of Shrinkage Stresses in Teeth Restored With Ceramic Inlays. Oper Dent. 2016 Sep-Oct;41(5):491-500 . doi: 10.2341/15-090-L.

12. Svizero Nda R, Silva MS, Alonso RC, Rodrigues FP, Hipolito VD, Carvalho RM, et al. Effects of curing protocols on fluid kinetics and hardness of resin cements. Dent Mater J. 2013;32(1):32-41.

13. Fonseca RG, Santos JG, Adabo GL. Influence of activation modes on diametral tensile strength of dual-curing resin cements. Braz Oral Res. 2005;19(4):267-71.

14. Kingman A, Hyman J, Masten SA, Jayaram B, Smith C, Eichmiller F, et al. Bisphenol $A$ and other compounds in human saliva and urine associated with the placement of composite restorations. J Am Dent Assoc. 2012;143(12):1292-302.

15. Lodiene G, Kopperud HM, Orstavik D, Bruzell EM. Detection of leachables and cytotoxicity after exposure to methacrylate- and epoxybased root canal sealers in vitro. Eur J Oral Sci. 2013;121(5):488-96.

16. Kwon HJ, Oh YJ, Jang JH, Park JE, Hwang KS, Park YD. The effect of polymerization conditions on the amounts of unreacted monomer and bisphenol A in dental composite resins. Dent Mater J. 2015;34(3):32735.

17. Park J, Eslick J, Ye Q, Misra A, Spencer P. The influence of chemical structure on the properties in methacrylate-based dentin adhesives. Dent Mater. 2011;27(11):1086-93.

18. Ferracane JL. Hygroscopic and hydrolytic effects in dental polymer networks. Dent Mater. 2006;22(3):211-22.

19. Pegoraro TA, da Silva NR, Carvalho RM. Cements for use in esthetic dentistry. Dent Clin North Am. 2007;51(2):453-71,x.

20. Heintze SD, Zimmerli B. Relevance of in vitro tests of adhesive and composite dental materials, a review in 3 parts. Part 1: Approval requirements and standardized testing of composite materials according to ISO specifications. Schweiz Monatsschr Zahnmed. 2011;121(9):80416.

21. Marghalani HY. Sorption and solubility characteristics of self-adhesive resin cements. Dent Mater. 2012;28(10):e187-98.

22. Faria-e-Silva AL, Piva E, Lima GS, Boaro LC, Braga RR, Martins LR. Effect of immediate and delayed light activation on the mechanical properties and degree of conversion in dual-cured resin cements. J Oral Sci. 2012;54(3):261-6.

23. Pereira SG, Fulgencio R, Nunes TG, Toledano M, Osorio R, Carvalho RM. Effect of curing protocol on the polymerization of dual-cured resin cements. Dent Mater. 2010;26(7):710-8.

24. Ozcan M, Zamboniota S, Valandro F, Bottino M, Bagis B. Microhardness of dual-polymerized resin cement around a translucent fiber post in the intraradicular environment. J Conserv Dent. 2011;14(4):370-3.

25. Sideridou ID, Achilias DS, Karabela MM. Sorption kinetics of ethanol/ 
water solution by dimethacrylate-based dental resins and resin composites. J Biomed Mater Res B Appl Biomater. 2007;81(1):207-18.

26. Malacarne J, Carvalho RM, de Goes MF, Svizero N, Pashley DH, Tay $\mathrm{FR}$, et al. Water sorption/solubility of dental adhesive resins. Dent Mater. 2006;22(10):973-80.

27. Malacarne-Zanon J, Pashley DH, Agee KA, Foulger S, Alves MC, Breschi $\mathrm{L}$, et al. Effects of ethanol addition on the water sorption/ solubility and percent conversion of comonomers in model dental adhesives. Dent Mater. 2009;25(10):1275-84.
28. Cekic-Nagas I, Ergun G. Effect of different light curing methods on mechanical and physical properties of resin-cements polymerized through ceramic discs. J Appl Oral Sci. 2011;19(4):403-12.

29. Giraldez I, Ceballos L, Garrido MA, Rodriguez J. Early hardness of self-adhesive resin cements cured under indirect resin composite restorations. J Esthet Restor Dent. 2011;23(2):116-24.

30. Yan YL, Kim YK, Kim KH, Kwon TY. Changes in degree of conversion and microhardness of dental resin cements. Oper Dent. 2010;35(2):203-10. 